\title{
A Design of the Model for Smart Home Gateway
}

\author{
Yang Deguo \\ School of Software \\ Shenyang University of Technology \\ Shenyang,China, 110023 \\ yangdg@sut.edu.cn
}

\author{
Zhang Honglei \\ Shenyang Command College of the Chinese Armed \\ Police Forces \\ Shenyang,China, 110113 \\ fzfln@sina.com
}

\begin{abstract}
A gateway is needed to control the smart devices at home. However, how to maintain or obtain the devices and form a mesh or tree shape topologies is still a question. There should be a model to form the architecture for the smart home objects. In this paper a multi-hop routing mechanism is proposed for smart home object and the algorithms are put forward for the smart objects to send and forward data packages to the gateway. The experiments show that the routing algorithm is practical and suitable for the home network.
\end{abstract}

Keywords- multi-hop, routing, gateway, 6LoWPANS

\section{INTRODUCTION}

A smart house is a house that has highly advanced automatic systems for lighting, temperature control, multimedia, security, window and door operations and many other functions [1].

A smart home appears intelligent because its computer systems can monitor so many aspects of daily living with low power sensors and other smart objects. As an important part of the smart home, the interconnection of WSN to the Internet has been widely researched in the last few years. At the beginning of the WSN era, researchers focused on the development of dedicated systems, where highly specialized but non-standard protocols were used within the WSN, whereas one or multiple gateways were used to translate messages and ultimately connect the WSN to the external IP world. While these systems were generally efficient in the special application scenario they were designed for, they are not flexible, and developing new applications on top of them was therefore time-consuming and cumbersome, as it required modifying the specialized protocols within the WSN. As a remedy to that, the 6LoWPAN standard has recently been proposed as a viable method to bring IPv6 to WSN [2][3] so that sensor nodes can be natively addressed and connected through the IP protocol. This has obvious advantages such as rapid connectivity and compatibility with preexisting architectures, plug-and-play installation of WSN, rapid development of applications as well as the possibility of integrating with existing services developed for standard IP networks.

6LoWPAN(IPv6 over Low power Wireless Personal Area Network)[3] are used for devices that are compatible with the IEEE 802.15.4 standard. Most of the LoWPAN devices are with low bandwidth, scarce memory capacity, short range, limited processing capability and other attributes of embeded hardware. Some nodes may acts as a host only, but other nodes may take part in routing. These host/routers are related with the processing and storage capabilities of the device and power available. IEEE 802.15.4 networks support star and mesh topologies. However, neither the IEEE 802.15.4 standard nor the 6LoWPAN format specification defines how mesh topologies for home network to be formed and maintained. Thus, 6LoWPAN formation and multi-hop routing can be supported either the logic link control layer or the IP layer. Because home smart objects nodes have special types and roles varies of energy, processing capabilities and so on, the method used in the ad-hoc networks is not suitable for home smart network, for the smart objects in the home migth stay in sleeping state for the most time. The tradional IP routing protocol used in Internet network is not customed for the home network either. Although a number of IP routing protocols have been developed in much work, these existing routing protocols could not satisfy the requirements of multi-hop routing in 6LoWPANs.

The document of RFC4919[5] bring about four requirements for 6 LoWPAN on routing protocols:

- low overhead on data packets.

- low routing overhead.

- minimal memory and computation requirements.

- support for sleeping nodes considering battery saving.

These four high-level requirements describe the basic requirements for 6LoWPAN routing.

Considering the problems of smart home, detailed 6LoWPAN routing requirements must be defined. The special application features of smart home affect the design of the routing requirements and the corresponding solutions. In this paper a multi-hop routing mechanism is proposed for smart home object and the algorithms are put forward for smart objects to send or forward the data package to the home gateway.

\section{The ARChitecture OF SMART Home Networks}

Smart home includes three parts, i.e the access point, the smart home networks and applications. The access point referred to as home gateway and the provision of services are distributed in the networks. The network refers to the cables which we already have to a large extent, both inside and outside the house, such as telephone cable, TV cable and the power supply network. It also refers to the extra infrastructure which in most cases does not exist yet: computer cables and lower voltage cables. It refers to infrared and radio frequent communication. 


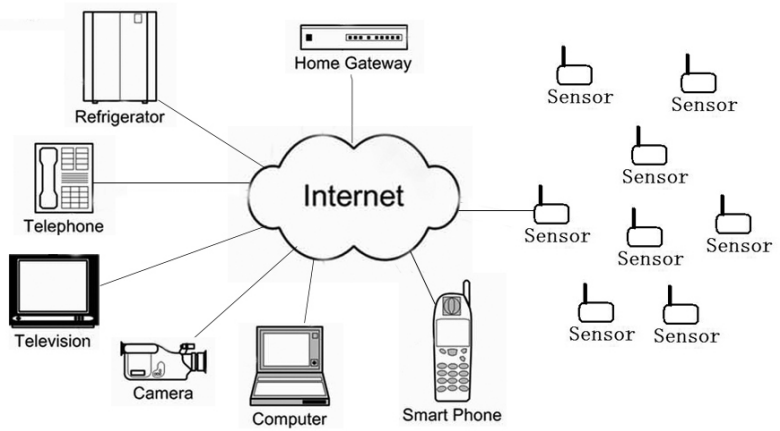

Figure 1. The model of home gataway

A smart home should be able to support the interaction of heterogeneous devices, networks. There are two sort of form of network, one is fixed wired network, and the other is wireless network. In smart homes, if a backbone fixed infrastructure is available then the fixed wired network may be better than an ad hoc system[6], and the mobile devices connected through wireless links to a fixed wired network. For instance the backbone network is based on the IP protocol, a robust and contrasted solution, which has demonstrated its success in the interconnection of heterogeneous devices. Most devices can be connected through this IP wired network, it will be simpler, and some other devices (e.g. sensors) may be connected using wireless IP communications, at the same time some device should be connected with the gateway by serial port, usb port and so on. In this case, a gateway is used to interconnect IP and nonIP sub-networks. This solution permits environmental control in a remote mode via a mobile as well as direct Internet access in home automation through residential gateways.

A typical example of this architecture is shown as Fig.1. There should be a home gateway which is connected with the parts controling the home devices and a sensor network to gathering the environment information in the home such as temperature, humidity, light intensity, pressure and so on. Mobile with intelligent operation system is used to control and monitor the home by home gateway, while the infrared remote control can be used at home. Some of the sensors may use a low-rate WPAN like 802.15.4[7]. Others may be integrated into the surroundings, probably connected through a backbone network like $802.11 \mathrm{~g}$. Some of the sensors can act as a bridge for the other sensor, providing interconnectivity at the network level. But additionally, these devices may interoperate themselves at a higher level. These networks have been designed very specifically for mobile device and optimized for low power usage. Bluetooth is currently the most widely used but its protocols are relatively complex and its power requirements are not suitable for devices that have to run on a small battery for years. Zigbee is a new type of very low power, low complexity network and it seems to be more suitable for the smart home applications.

\section{THE Design OF THE ModeL}

In this section, we define the home gateway model based on the followed assumptions that part of the device is with power, and can be directly connected with home intelligent gateway, and moreover, some of the devices do not have the IP address, such as remote control, cameras, refrigerators, mobile with wireless mobile networks and some other equipment connected to relay control. The others of the devices have IP address, such as various sensors, and sensor base station board, in particular, the home gateway.

Because some of the device can only use the batterypowered, such as sensors in the house, and these devices are energy constrained devices, in this model, their energy should be considered as a parameter of its capability.

In this paper, some smart objects of the home gateway can only be easily connected with gateway through the serial port, USB port or RJ45 interface, such as cameras, smart relays and so on. It is not diffcult to to achieve, and is not descripted in detail in this paper. While the sonsers used in the smart home has its characters of energy-constrained and it may be routed by other device, so it is complex to form the contact with the gateway, so we will emphasis on the sensor network used in the smart home.

As part of the home gateway, the sensor networks is different from ordinary ad hoc networks, for the sensors at home is in a fixed position, the distant is not far away with each other, its data will be passed to the home gateway finally, and the home gateway is fixed position too. So we need to create a new efficient algorithm to let the entire sensor establish a network, which is suitable for the smart home.

Because the capability of the sensors is important for forming network, we should take the factor of its energy and hops into account. We defined the capability from one sensor to the other as the measuredvalue, and the measuredvalue is calculated according to the following methods:

If the hop between the sensor and gateway is $\boldsymbol{h}$, and its energy is $\boldsymbol{e}$, then

measuredvalue $=\mathrm{h} \times\left(2^{E / e}\right)$

The $\boldsymbol{e}$ is the percent of it full energy $\boldsymbol{E}$. When the energy on the sensor is disspating gradually, the measuredvalue will become infinite, and the node would not be choosen by the others to forward the data package.

In order to save energy for each sensor, we use a protocol like RIP(routing information protocol) to route data for each other. Each sensor will broadcast its information to the nodes which it can connect, the information include the number of hops to the destination home gateway and its remaining energy. Each sensor will caculate the measuredvalue and updates its mini-routing table based on the received information. When a sensor will send data of itself or from others, it will select the sensor whose measureedvalue is the least as the next hop, and transfer the data to it. To avoid the question of loops, before each sensor send the data, it will add its ID in the head of the data. When a sensor found an ID in the head of the date, it will not forward the data package to the sensor. 
The algorithm is shown in Table I .

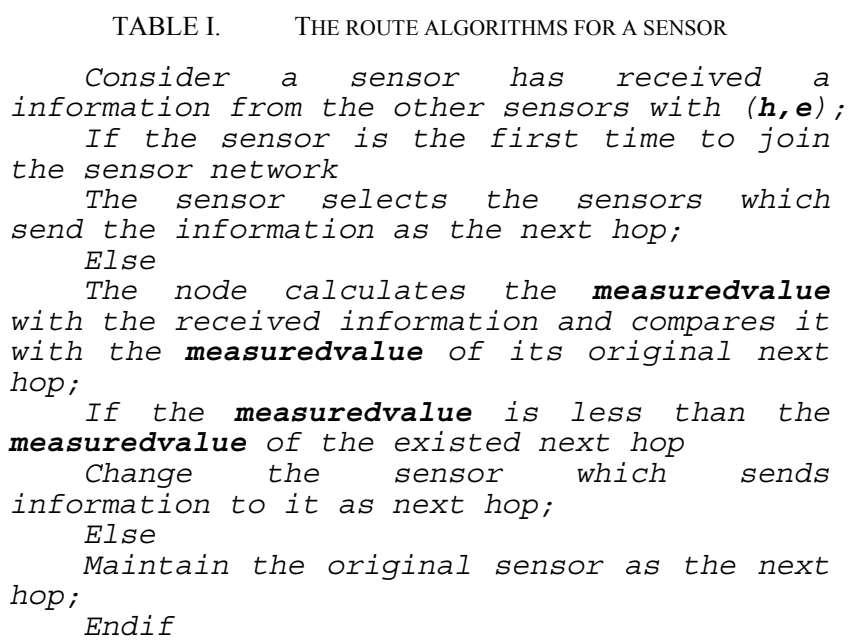

\section{EXPERIMENT RESULT AND ANALYSIS}

In this section we investigate the performance of the home gateway model by conducting experiments.

An ARM11-based smart home system of wireless sensor are designed and implemented. The system combines ARM11 intelligent terminals with wireless sensor networks, camera, relay controller, GSM modules and so on. Qt is used to design the interface, and it is beautiful and with the feature of easy operation. NesC is used to design part of wireless sensor networks. The programming ideas based on TinyOS simplifies the development process. The Camera is used in the system too, and the quality of image can meet the monitoring requirements. Wireless sensor networks, relay controller, GSM modules with RS232 cable and ARM11 are intelligent terminal connected, and the system is scalable. The system is shown in Figure 2.

The embedded ARM11 device is from Beijing BoChuang Technology Co., which is used as the home gateway, and it is equipped with three serial interfaces, one network RJ45 port, four USB ports and one audio interface. The sensor network devices are form Emdoor Electronic Technology Co., which includes one sensor base station board connected with the home gateway by serial interfaces, and eight Temperature and humidity sensors, four photosensitive sensors, three flame sensors and one flow sensor. All the sensors are distributed in different rooms and outdoor. The camera is connected with the gateway by the USB port. The relay control module and GSM module are connected with the serial too. The gateway is connected with the Internet, and it is with Linux kernel, QT GUI and JFFS2 file system. We develop the server on the gateway and a client on a mobile with Android operating system. The gateway can control the smart device by relay and collect information from the sensor network and camera. The mobile can control the device in the home and read the information through the gateway. The sensor routing algorithm designed above is used, and the sensor broadcast it routing information every five minutes. The result is we can control the home smart object by mobile, and the sensor network worked well. The average delay time from the mobile to the server is $210 \mathrm{~ms}$, for the mobile will connect the home gateway through mobile network and then through IP network.

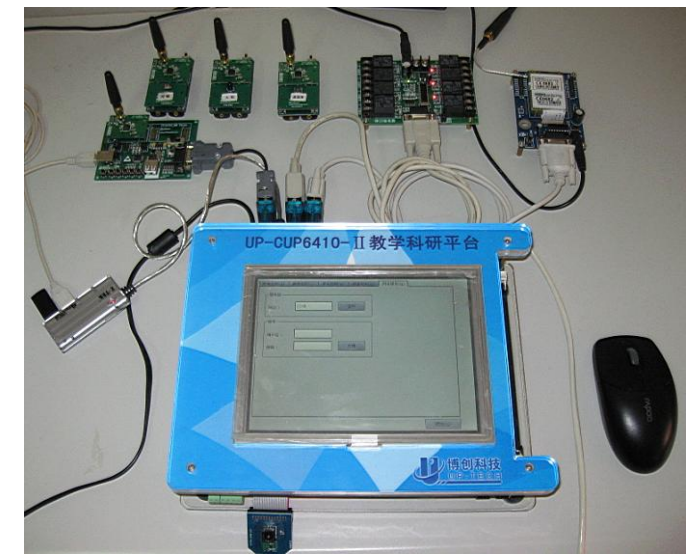

Figure 2. The home gateway system based on sensors network

\section{SUMMARIES}

A smart home gateway model is designed in this paper, in the model, a mobile is used to control the device and collect home information in the home through home gateway. Because the gateway is connected with a sensor network, a simple routing algorithm for the home sensor is put forward. The experiment showed that the design is effective and feasible.

The future work includes two sides. One, more flexible application should be developed and deployed on the home gateways, the other, it is should be tested that how often the routing information is broadcasted will achieve the best performance, and the parameter should be decided in the practice.

\section{REFERENCES}

[1] Guy Dewsbury; Bruce Taylor; Martin Edge, The Process of Designing Appropriate Smart Homes, Conference or Workshop Item (UNSPECIFIED), 2001, PP 131-146.

[2] A. Dunkels and J. P. Vasseur, IP for Smart Objects, IPSO Alliance White Paper No. 1, Sept. 2008.

[3] ] J. W. Hui and D. E. Culler, IP is Dead, Long Live IP for Wireless Sensor Networks, in Proc. of ACM SenSys, Nov. 2008.

[4] J. Hui; P. Thubert, Compression Format for IPv6 Datagrams in Low Power and Lossy Networks (6LoWPAN), RFC draft, http://www.ietf.org/id/draft-ietf-6lowpan-hc-15.txt.

[5] N. Kushalnagar; G. Montenegro, IPv6 over Low-Power Wireless Personal Area Networks (6LoWPANs): Overview, Assumptions, Problem Statement, and Goals. RFC draft, http://datatracker.ietf.org/doc/rfc4919.

[6] Benyuan Liu; Don Towsley, Capacity of a wireless ad hoc network with infrastructure, MobiHoc '07 Proceedings of the 8th ACM international symposium on Mobile ad hoc networking and computing, ACM New York, NY, USA, 2007.

[7] Howitt, I.; Gutierrez, J.A., IEEE 802.15.4 low rate - wireless personal area network coexistence issues[J]. Wireless Communications and Networking, 2003(WCNC 2003). PP 1481 1486 vol.3. 